\title{
RESEÑA
}

\section{Santiago Renjifo Salcedo \\ 1913 - 1965}

Los Panidas eran trece, los Pentateucos eran cinco; dos grupos creados más o menos en la misma época; uno de artistas, el otro de científicos naturalistas. Dos conglomerados de hombres pulcros, generosos e ilustres que brillaron en el ámbito nacional e internacional.

Una semblanza de Santiago Rengifo, con ocasión de los ochenta años de la fundación del instituto Nacional de Salud, debe enmarcarse dentro del grupo llamado la Sociedad Biológica del Pentateuco, conformada por cinco miembros, cada uno de los cuales, en una u otra forma, contribuyó a los logros de la institución.

Para comprender el espíritu que guió a sus miembros, César Uribe Piedrahita, Ernesto Osorno, Hernando Osorno, Santiago Renjifo Salcedo y Guillermo Varela, me permitiré transcribir el acta inaugural de la Sociedad Biológica del Pentateuco, llevada a cabo en El Arrayán, hoya hidrográfica del río San Francisco, entre los cerros de Monserrate y Guadalupe, a 3.300 metros sobre el nivel del mar, municipio de Bogotá.

Se organiza esta sociedad hoy 23 de agosto de 1944, con el objeto de hacer progresar la investigación de nuestra flora y fauna regionales. Por lo tanto, los miembros que la integran, en número de cinco, emprenderán esta clase de estudios. La sociedad se denomina PENTATEUCO con el fin de desertar cualquier principio atávico que no esté sometido a una sana discusión e interpretación y en ella se desechará toda cuestión religiosa, política y aun de amistad, con el fin de hacer crítica científica justa e imparcial.

Nos reunimos en el monte, siguiendo el aforismo de uno de nosotros que no es el caso de nombrar: "La ciencia no tiene templo".

De acuerdo con estas reflexiones, elaboraremos un reglamento que aparentemente será muy estricto porque se nota que la ciencia en nuestro medio está adquiriendo caracteres de simulación y rabuleyismo.

En esta sociedad sui generis sorprenderá uno de los artículos del reglamento: "El presidente será elegido a cara o sello". Sabia elección para prescindir de chismes infinitesimales que nunca nos deben tocar.

El secretario será a su vez tesorero perpetuo, porque quien es capaz de escribir fielmente nuestras discusiones, es también el individuo decoroso para conservar íntegramente el vil metal.

Ningún miembro se resentirá si uno o más de los otros le enfrenta sus baterías en cualquier tema científico.

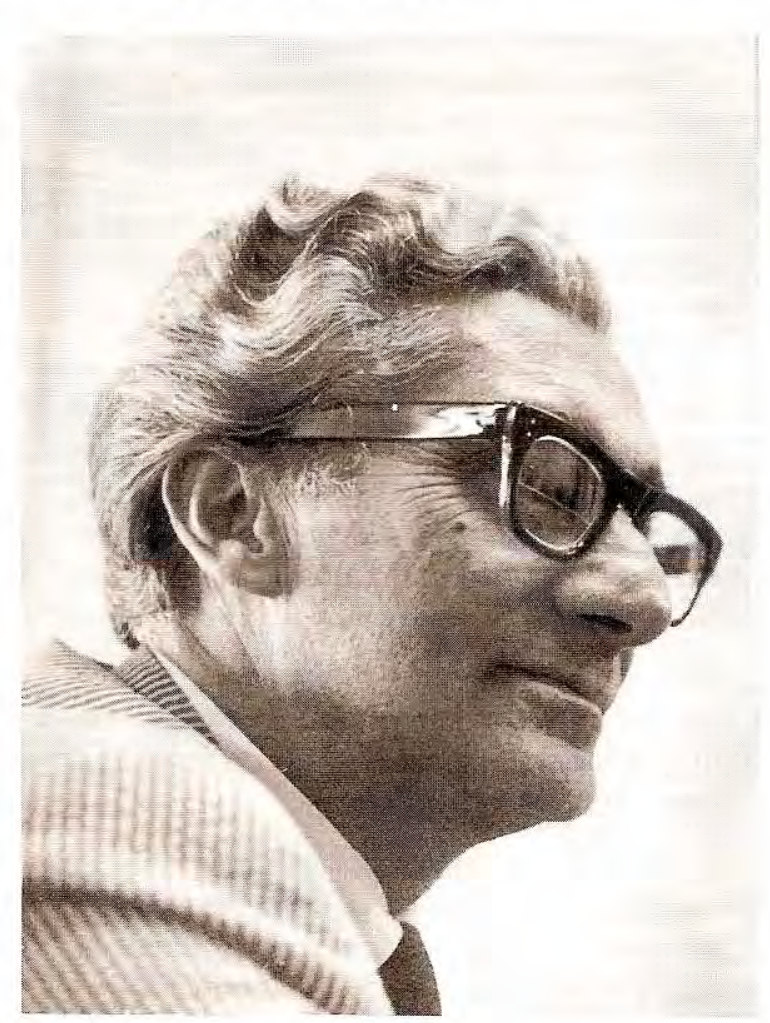


Como es difícil, en ocasiones, prescindir de ciertos prejuicios, la presente sociedad, por razones esencialmente prácticas impone la norma que hizo grande a Persia: "La lucha del principio del bien y el principio del mal." Aquí sentaremos bases para hacer progresar el principio que lógicamente más nos beneficie.

La conducta que nos debe guiar en nuestras excursiones es aquella que se ajuste a la mejor protección de las especies, al evitar en cuanto sea posible la destrucción brutal de la morada de la fauna; por tanto, basados en el reciente hallazgo de dos especies de ranas vernáculas de esta área, de difícil consecución, socio que levante una piedra, la debe dejar en la misma posición en la que la encontró, pues de lo contrario, los bichos no hallarán su albergue, preparado quizá desde mucho tiempo atrás.

En esta sociedad sin fundador, consideramos superfluo el famoso curriculum vitae, porque los miembros que la integran ya tienen su hoja de servicios conocida que, indefectiblemente, continuarán perfeccionando en este cenáculo en donde cada uno será estimulado por los otros, dentro de la más estricta ecuanimidad.

En nuestra sociedad es permitido, tolerable y necesario el chiste, principal diferencia entre el naturalista investigador y sus súbditos (los animales y las plantas).

Ningún miembro de nuestra sociedad tiene remplazo, de modo que se irá extinguiendo como sucede con los Bombus rubicundus y otras especies de melíferos sociables comunes en esta zona, cuando muere el último abejorro obrero.

Esta región de El Arrayán es algo conocida por dos de los miembros del Pentateuco. Tiene grandes puntos incitativos de investigación, como la biología de:

A) las salamandras del género Edipus, carente de pulmones y denominado así por su aparente ceguera y que el fundador de este género relacionó con el famoso rey de

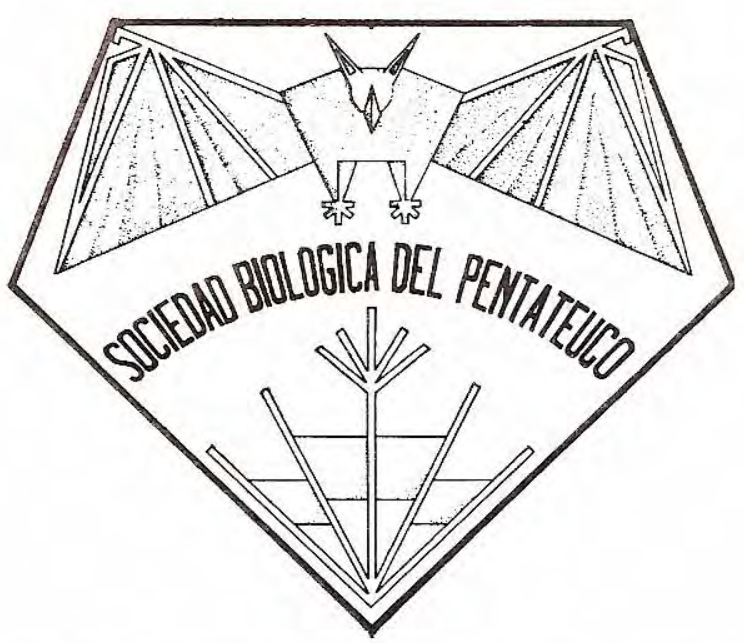

Tebas, de la mitología griega, quien después de arrancarse los ojos, huyó guiado por su hija Antígona;

B) la nueva especie Ixodes montoyanus Cooley (Acarina: Ixodeides) parásito del "guache", Nasulla olivacea.

De las especies Anopheles (Lophodomyia) o lketoracras Osorno (Diptera: Culicidae) que representa el registro de mayor altura hasta ahora conocido para este género.

Nuestro símbolo heráldico se basa en la interesante ecología de las bromelias, plantas llamadas vulgarmente "quiches" en esta región, "vicundos" en Nariño, "carros" en el Valle, que representan según Picado, autor de una obra sobre bromelias en Costa Rica, los mares aéreos, concepción para llenar grandes espíritus con investigaciones biológicas.

Sesionaremos escogiendo el sitio apropiado y al calor de una bebida alcohólica tradicional (vino de consagrar), no con pretexto religioso y sin rendir culto excesivo a Baco.

Nunca reinará la indiferencia entre nosotros, de modo que cualquier idea será discutida, rechazada o consignada en los archivos para que vaya quedando historia de nuestras sinceras aficiones.

Queda así constituida la Sociedad Biológica del Pentateuco, cuyo reglamento se consig- 
nará en papel de música, con artículos pentagramales en próximas sesiones de fecha imprevisibles. El Arrayán, Cerro de Monserrate, Bogotá,
1944.

Hernando Osorno, Ernesto Osorno, César Uribe Piedrahita, Santiago Renjifo Salcedo, Guillermo Varela.

Los miembros de la sociedad se fueron extinguiendo, comenzando por César Uribe Piedrahita y terminando con Guillermo Varela, el gran artista, ilustrador científico de muchos de los trabajos de los otros miembros.

Santiago Renjifo Salcedo nació en la ciudad de Buga el 31 de diciembre de 1913; sus estudios de primaria y secundaria los realizó en los colegios Villegas y Académico de Buga y sus estudios universitarios en la Universidad Nacional de Colombia donde se gradúo el 18 de abril de 1944 después de haber ejercido con brillantez los cargos de preparador del Laboratorio de Parasitología, concurso de 1940; médico jefe de sanidad en Puerto Merizalde, Yurumanguí, Cajambre y Naya, 1942-1943; médico entomólogo asistente de la comisión de estudios sobre bartonelosis en Nariño y Cauca, 1943; médico jefe de la comisión vacunadora No.2 contra la fiebre amarilla, 1943-1944.

Aunque la dedicatoria de su tesis no figura en el acta de grado, titulada Notas entomológicas regionales, realizada en el Laboratorio de Entomología de la Sección de Estudios Especiales del Ministerio de Trabajo, Higiene y Previsión Social, bajo la supervisión del doctor Ernesto Osorno Mesa, está dedicada "A la negra María, canción del Africa lejana, errante por los manglares del Pacífico". Dedicatoria que muestra algo que caracterizó a Santiago: su sentido poético y musical. Cantante en los coros de las iglesias de Bogotá en su época de estudiante, que ayudaban al bien comer y, posteriormente, acompañante de Miguel Angel Martín (EI Cojo), en su querido llano, tocando el tiple.

En 1945, obtuvo el título de Master of Public Health de la Universidad de Johns Hopkins, de Baltimore, Maryland.
Durante 1947, a su regreso de Norteamérica, se desempeñó como Director de la División de Enfermedades Comunicables del Ministerio de $\mathrm{Hi}$ giene y Jefe de Investigaciones de Malaria.

De 1948 a 1950, se desempeñó como Director del Instituto de Enfermedades Tropicales Roberto Franco de Villavicencio, instituto dependiente de la Sección de Estudios Especiales del Ministerio de Higiene que, posteriormente, constituiría el Instituto de Estudios Especiales Carlos Finlay.

Son estas instituciones las que admirablemente emprendieron estudios sobre enfermedades tropicales en forma prospectiva produciendo un sinnúmero de publicaciones de alta calidad científica y formando científicos que hoy pueden ser paradigma para las nuevas generaciones. Santiago Rengifo, entre muchos otros, es uno de ellos.

En 1952, regresó a su nativo Valle lleno de entusiasmo por crear la Escuela de Medicina de la Universidad del Valle, iniciativa que logró junto con otros colegas. En 1956, se fundó el Departamento de Medicina Preventiva, iniciando una nueva manera de enfocar los estudios médicos en el país. Dirigió este departamento hasta el año 1959, cuando la Universidad Nacional lo nombró como Director de la Escuela de Salud Publica la cual dirigió hasta el año 1962, cuando la mezquindad de algunos pocos, que no pudiendo resistir su grandeza, con las artimañas de la politiquería lo obligaron a renunciar.

En 1962, viajó al Africa como asesor de la Organización Mundial de la Salud en Brazzaville, República del Congo, desde donde lo llamó el presidente Guillermo León Valencia en 1963 para desempeñar el Ministerio de Salud Pública, cartera que regentó hasta 1965 cuando nuevamente fue llamado por la Organización Mundial de la Salud para ocupar la jefatura de la zona $V$ de América con sede en Rio de Janeiro. La noche del 28 de diciembre lo vi por última vez; el 30 me llamaron a las 6 a.m. para informarme que Santiago había muerto en un absurdo accidente de tránsito. Como el pico que lleva su nombre en la sierra de La Macarena, región que el amó, su memoria seguirá siendo un hito para todos aquellos que se dediquen a la medicina preventiva, a la salud pública o al humanismo. 
La revista Cespedesiaen su volumen III, números 9-12, de enero a diciembre de 1974, está totalmente dedicada a la recopilación de los trabajos de Santiago Renjifo Salcedo hecha por el doctor Pablo Barreto, quien fuera su compañero de trabajo y su amigo dilecto en la Universidad del valle.

\section{Contribuciones a la entiomología colombiana}

Renjifo S. Notas entomológicas regionales. Trabajo presentado para optar al título en medicina y cirugía. Cali: Gráficas Palásquez; 1944.

Renijo S. Eratyrus cuspidatus Stal en Colombia. Bogotá: Rev Laboratorio 1945; No. 18.

Renjifo S, Patiño VM. Contribución al conocimiento de la entomofauna médica del bajo Calima: presentación del Calima. Cali; 1946:1-63.

Renjifo S. Disecciones de mosquitos del género Anopheles en la intendencia del Meta. Caldasia 1948;V(20):99103.

\section{Contribuciones a la parasitología colombiana}

Renjifo S. Parásitos de quirópteros de los Llanos Orientales. Anales de la Sociedad de Biología de Bogotá 1948; $/ 11(3): 98-101$.

Renjifo S. Mansonella ozzardi en la región oriental de Colombia. Anales de la Sociedad de Biología de Bogotá 1949 ; III(5):211-6.

Renjifo S. Informe sobre actividades del Instituto de Enfermedades Tropicales Roberto Franco de mayo de 1948 a mayo de 1949. Bogotá: Memorias del Ministerio de Higiene 1949;2:163-83.

Renjifo S. Memorandum sobre la sierra de La Macarena y estación biológica José Jerónimo Triana. Rev Fac Nal Agronomía (Medellín) 1949;IX(35).

Renjifo S. Hemopárasitos de aves y otros vertebrados de los Llanos Orientales. Anales de la Sociedad de Biología de Bogotá 1950;IV(1).

Renjifo S. Enseñanza de la medicina preventiva y salud pública en la Facultad de la Universidad del Valle, Cali, Colombia. Bol Oficina Sanit Panam 1959;XLVII(5):401-11.

Gast A, Renjifo S. Leishmaniosis visceral. Estudio epidemiológico del primer caso diagnosticado en Colombia. Anales de la Sociedad de Biología de Bogotá 1944;L(4):1-8.

Bequaert J, Renjifo S. The tabanidae of Colombia (Diptera). Psyche 1964;53:52-86.
Renjifo S, Osorno E. Presencia de Ornithodoros furcosus Neumann en Imués, Nariño, y notas sobre el género Ornithodoros en Colombia (Acarina: Ixodoidea); 1948.

Renjifo S, Osorno E. Dasypus novemcintus, procedente de Ocoa, Villavicencio, Meta, naturalmente infectado con Trypanosoma cruzi Chagas. Revista de la Academia Colombiana de Ciencias Exactas, Físico-Químicas y Naturales, 1950; VII(28):548-50.

Renjifo S, Orduz A. Dieciocho nuevos casos humanos, autóctonos, con Mansonella ozzardi en el oriente de Colombia. Revista de la Academia Colombiana de Ciencias Exactas, Físico-Químicas y Naturales 1950; VII(28): 548.

Renjifo S, Piedrahita C, Groot H. Contribución de tripanosomas humanos y de animales en Colombia. Revista de Higiene Bogotá 1950;24:3.

Rey $\mathrm{H}$, Renjifo S. Anopheles (N). Nuñez-Tovari infestado en la naturaleza con Plasmodium sp. Revista de la Academia Colombiana de Ciencias Exactas, Físico-Químicas y Naturales 1950; VII(28):534-40.

Groot H, Renjifo S, Piedrahita C. Nuevos datos sobre Trypanosoma ariari. Anales de la Sociedad de Biología de Bogotá 1950;4(3):1950:81-4.

Groot H, Renjifo S, Piedrahita C. Nota preliminar sobre inoculación a un voluntario con Trypanosoma sp. (ariari). Anales de la Sociedad de Biología de Bogotá 1950;4(3):99-103.

Groot H, Renjifo S, Piedrahita C. Algunas consideraciones sobre el llamado Trypanosoma rangeli. Anales de la Sociedad de Biología de Bogotá 1951;4(6):225-30.

Groot H, Renjifo S, Piedrahita C. Trypanosoma ariarin. sp., from man, found in Colombia. Am J Trop Med 1951;31(6):673-91.

Renjifo S, de Zulueta J. Five years observations of rural malaria in eastern Colombia. Am J Trop Med 1951;31(4): 598-611.

Renjifo S, Sanmartín C, Zulueta J. A survey of the blood parasites of vertebrates in eastern Colombia. Acta Trop Revue de Sciences Tropicales et Médecine Tropicale 1952;IX(2):151-69.

Groot H, Osorno E, Renjifo S. Anotaciones sobre el problema de las tripanosomiasis humanas en Colombia. Memorias, Primer Congreso Interamericano de Higiene, La Habana, Cuba; 1953:714-9. 